\title{
The Distribution of Crossings of Chords Joining Pairs of $2 n$ Points on a Circle
}

\author{
By John Riordan
}

\begin{abstract}
This paper, in the first place, calls attention to an extraordinarily compact solution of the problem in the title, given (a trifle hidden) in the work of the late Jacques Touchard. Its main weight, however, is on properties of the several kinds of number sequences appearing.
\end{abstract}

1. Introduction. Alfred Errera [1] apparently was the first to pose and solve the problem: in how many ways may $2 n$ points on a circle be joined in pairs so that the corresponding chords do not intersect within the circle. The answer is what I am accustomed to call the Catalan number, $c_{n}=(2 n) ! / n !(n+1) !$, which is also associated with ballots and parenthesizations. More recently the brothers Yaglom [9, Problem 54] have re-examined the problem and supplied a mapping onto ballots. The problem has the natural extension to the enumeration of all possible $(1 \cdot 3 \cdots(2 n-1))$ pairings of $2 n$ points on a circle by number of crossings of the chords.

This extension was put to me by a friend, G. W. Ford, because of its interest (actually only tangential) in the combinatorial approach to the Ising model initiated by M. Kac and J. C. Ward [2] . Sometime much later, I found that an extraordinary compact solution had been given by the late Jacques Touchard [6], [7], [8] .

This is as follows. With $T_{n}(x)$ the enumerating generating function by number of crossings, then

$$
t_{n}(x)=(1-x)^{n} T_{n}(x)=\sum_{0}^{n}(-1)^{j} t_{n j} x^{J}, \quad J=\left(\begin{array}{c}
j+1 \\
2
\end{array}\right)
$$

with

$$
\begin{aligned}
t_{n j} & =a_{n+j, n-j}=\left(\begin{array}{c}
2 n \\
n-j
\end{array}\right)-\left(\begin{array}{c}
2 n \\
n-j-1
\end{array}\right) \\
& =\frac{2 j+1}{j+n+1}\left(\begin{array}{c}
2 n \\
n-j
\end{array}\right)=\frac{2 j+1}{2 n+1}\left(\begin{array}{c}
2 n+1 \\
n-j
\end{array}\right) .
\end{aligned}
$$

The number $a_{n m}$ is the number of weak-lead election returns with final vote $(n, m)$ and $n \geqslant m$, that is, the number of lattice paths from $(0,0)$ to $(n, m)$ without

Received February 21, 1974.

AMS (MOS) subject classifications (1970). Primary 05A15; Secondary 10A35.

Key words and phrases. Catalan, ballot numbers (numbers of Delannoy), generating functions, recurrence, number sequences.

Copyright $\odot 1975$, American Mathematical Society 
crossings of diagonal points $(k, k) ; a_{n 0}=1, a_{n m}=a_{n, m-1}+a_{n-1, m}, m=$ $1(1) \overline{n-1}$ and $a_{n n}=a_{n, n-1}=c_{n}$, the Catalan number. Touchard calls them numbers of Delannoy, with a reference to [1]. It may be noted at once that their recurrence implies (cf. [4, p. 661)

$$
\begin{aligned}
t_{n, 0} & =t_{n-1,0}+t_{n-1,1}=c_{n}, \\
t_{n, j} & =t_{n-1, j-1}+2 t_{n-1, j}+t_{n-1, j+1}, \quad j=1(1) n,
\end{aligned}
$$

and also $\left(\delta_{n m}\right.$ is the Kronecker delta here and later)

$$
\begin{gathered}
t_{n, 0}+t_{n 1}+\cdots+t_{n n}=\left(\begin{array}{c}
2 n \\
n
\end{array}\right), \\
t_{n}(1)=t_{n 0}-t_{n 1}+\cdots+(-1)^{n} t_{n n}=\delta_{n 0} .
\end{gathered}
$$

The table for $t_{n j}$ starts as follows:

\begin{tabular}{rrrrrrr}
\hline$j / n$ & 0 & 1 & 2 & 3 & \multicolumn{1}{c}{4} & 5 \\
\hline 0 & 1 & 1 & 2 & 5 & 14 & 42 \\
1 & & 1 & 3 & 9 & 28 & 90 \\
2 & & & 1 & 5 & 20 & 75 \\
3 & & & & 1 & 7 & 35 \\
4 & & & & & 1 & 9 \\
5 & & & & & & 1
\end{tabular}

Because Eq. (1), b̧y a strange oversight, does not appear explicitly in any of the Touchard references cited, I give a short derivation in the next section, using results in [8]. This is followed by an examination of the immediate implications of (1) on the coefficients $T_{n k}$ of $T_{n}(x)$, and by a similar study of other Touchard identities.

I do not take space to prove the following results on the probability distribution $T_{n}(x) / T_{n}(1)$ : the mean is $n(n-1) / 6$ and the variance is $(n+3) n(n-1) / 45$.

2. The Touchard Formula for $T_{n}(x)$. In [6], Touchard arrived at the present problem in an effort to get more light on the frustrating enumeration of foldings of strips of postage stamps. The form it took is different from, but equivalent to, that described above and may be stated as follows. The $2 n$ points are uniformly distributed on a line, and are connected in pairs by $n$ convey arcs, all above the line; the double points on these arcs correspond to the crossings of chords on the circle. For illustration, for $n=2$, the diagrams for the three pairings: $(12)(34),(13)(24)$ and (14)(23) are
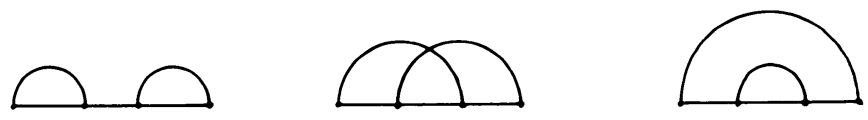

It is clear that the first of these falls into two parts, each the single arc for $n=1$ and may be indicated by $\left(1^{2}\right)$; the other two do not and are indicated (together) by (2). 
If $S_{1}(x), S_{2}(x)$ are the enumerators of these pairing classes by number of double points, then

$$
T_{2}(x)=\left[S_{1}(x)\right]^{2}+S_{2}(x)=1+(1+x)=2+x
$$

Similarly

$$
T_{3}(x)=\left[S_{1}(x)\right]^{3}+2 S_{2}(x) S_{1}(x)+S_{3}(x) .
$$

Thus, for general $n$, the terms appearing correspond to partitions of $n$, with numerical coefficients given by permutations of elements of character specified by the corresponding partition, and, abbreviating $S_{i}(x)$ to $S_{i}$,

$$
T_{n}(x)=\sum\left[k ; k_{1}, \cdots, k_{n}\right] S_{1}^{k_{1}} \cdots S_{n}^{k_{n}}
$$

with $n=k_{1}+2 k_{2}+\cdots+n k_{n}, k=k_{1}+k_{2}+\cdots+k_{n},\left[k ; k_{1}, \cdots, k_{n}\right]$ a multinomial coefficient and summation over all partitions of $n$. Equation (2) recalls the relations of elementary symmetric functions $a_{n}$ and homogeneous product sums $h_{n}$ (cf., e.g., [4, p. 188]) which follow from

$$
1=\left(1-a_{1} y+a_{2} y^{2}-a_{3} y^{3}+\cdots\right)\left(1+h_{1} y+h_{2} y^{2}+\cdots\right),
$$

and indeed (2) follows from

$$
1=\left(1-S_{1} y-S_{2} y^{2}-S_{3} y^{3}-\cdots\right)\left(1+T_{1} y+T_{2} y^{2}+\cdots\right)
$$

where as above $S_{i}$ is short for $S_{i}(x), T_{i}$ for $T_{i}(x)$.

In terms of the generating functions

$$
S(x, y)=\sum_{n=0} S_{n}(x) y^{n}, \quad T(x, y)=\sum_{0} T_{n}(x) y^{n}, \quad S_{0}(x)=T_{0}(x)=1,
$$

Eq. (3) has the form:

$$
1=(2-S(x, y)) T(x, y) .
$$

Now notice that Eq. (32) of [8], namely

$$
1-(1-x) z F(x,(1-x) z)=(1-z \phi(z)) / A(x, 1-\phi(z))
$$

with $A(x, y)=1+x y+x^{3} y^{2}+\cdots+x^{N} y^{n}+\cdots, N=\left(\begin{array}{c}n+1 \\ 2\end{array}\right)$, and (unnumbered equation following (20) of [8]), $F(x, z)=S_{1}(x)+S_{2}(x) z+\cdots+S_{n}(x) z^{n-1}+$ $\cdots$, while $\phi(z)=c(z)$, the generating function for Catalan numbers, $c_{n}$, may be rewritten

$$
2-S(x,(1-x) z)=(1-z c(z)) / A(x, 1-c(z))
$$

Hence, by (3a)

$$
T(x,(1-x) y)=A(x, 1-c(y)) /(1-y c(y))=c(y) A(x, 1-c(y)),
$$

since $1=c(y)(1-y c(y))$. Then, since $1-c(y)=-y c^{2}(y)$, Eq. (5) is the same as 


$$
\begin{aligned}
T(x,(1-x) y) & =\sum_{0} t_{n}(x) y^{n}=c(y) A\left(x,-y c^{2}(y)\right) \\
& =\sum_{j=0}(-1)^{j} x^{J} y^{j} c^{2 j+1}(y), \quad J=\left(\begin{array}{c}
j+1 \\
2
\end{array}\right) .
\end{aligned}
$$

But $c^{p+1}(y)=\Sigma_{0} a_{p+n, n} y^{n}$ (cf. [8, Eq. (6)], or [4, p. 153]); hence (1) follows by equating coefficients of $y^{n}$ in (6).

The equation $t_{n}(x)=(1-x)^{n} T_{n}(x)$ and its inverse $T_{n}(x)=(1-x)^{-n} t_{n}(x)$ of course imply identities in the coefficients $t_{n j}$ and $T_{n k}$ (the coefficient of $x^{k}$ in $\left.T_{n}(x)\right)$, namely

$$
\begin{aligned}
t_{n j} & =\sum_{0}(-1)^{j+k}\left(\begin{array}{l}
n \\
k
\end{array}\right) T_{n, J-k}, \quad J=\left(\begin{array}{c}
j+1 \\
2
\end{array}\right), \\
0 & =\sum_{0}(-1)^{k}\left(\begin{array}{l}
n \\
k
\end{array}\right) T_{m, m-k}, \quad m \neq j, j=1(1) n, \\
T_{n k} & =\sum_{0}(-1)^{j} t_{n j}\left(\begin{array}{c}
n+k-J-1 \\
n-1
\end{array}\right), \quad J=\left(\begin{array}{c}
j+1 \\
2
\end{array}\right) .
\end{aligned}
$$

From these, some of the $T_{n k}$ may be found explicitly. Thus $T_{n 0}=t_{n 0}=c_{n}$, in agreement with [1],

$$
\begin{aligned}
& T_{n 1}=n T_{n 0}-t_{n 1}=\left(\begin{array}{c}
2 n \\
n-2
\end{array}\right), \\
& T_{n 2}=n T_{n 1}-\left(\begin{array}{l}
n \\
2
\end{array}\right) T_{n 0}=\frac{1}{2}\left(\begin{array}{c}
n+3 \\
1
\end{array}\right)\left(\begin{array}{c}
2 n \\
n-3
\end{array}\right) .
\end{aligned}
$$

These results and the further expressions for $k=3(1) 6$ appear in $[6$, pp. 395 and 396] with the notation $U_{2 n}(k)$ for $T_{n k}$.

The polynomial $T_{n}(x)$ is of degree $N=\left(\begin{array}{l}n \\ 2\end{array}\right)$, and it is interesting to note that the relations above imply

$$
T_{n, N-j}=\left(\begin{array}{c}
n+j-1 \\
j
\end{array}\right), \quad j=0(1) \overline{n-1},
$$

because this result holds also for the corresponding coefficient of $J_{n+1}(x)$, the enumerator for labelled trees by number of inversions [3]. For concreteness, I repeat the table in [6], for $T_{n k}, n=1(1) 5, k=1(1) N, \quad N=\left(\begin{array}{c}n \\ 2\end{array}\right)$

\begin{tabular}{rrrrrrrrrrrr}
\hline$n / k$ & 0 & 1 & 2 & 3 & 4 & 5 & 6 & 7 & 8 & 9 & 10 \\
\hline 1 & 1 & & & & & & & & & & \\
2 & 2 & 1 & & & & & & & & & \\
3 & 5 & 6 & 3 & 1 & & & & & & & \\
4 & 14 & 28 & 28 & 20 & 10 & 4 & 1 & & & & \\
5 & 42 & 120 & 180 & 195 & 165 & 117 & 70 & 35 & 15 & 5 & 1
\end{tabular}


It is worth noting (because of its interest in the Ising model) that $t_{n}(-1)=$ $t_{n 0}+t_{n 1}-t_{n 2}-t_{n 3}+\cdots+(-1)^{[j / 2]} t_{n j}+\cdots+(1)^{[n / 2]} t_{n n}$. Using the recurrences for $t_{n j}$ given earlier, this implies

$$
t_{n}(-1)=2 t_{n-1}(-1)=2^{n}, \quad T_{n}(-1)=2^{-n} t_{n}(-1)=1 .
$$

3. Imaginary Arguments. In [8, p. 10] Touchard exhibits the remarkable formula

$$
S_{n}(i)=(1+i)(1+2 i)^{n-2}, \quad n=2,3, \cdots ; i^{2}=-1 .
$$

Setting $S_{n+2}(i)=a_{n}+i b_{n}$, it follows at once that

$$
a_{n+1}-a_{n}=-2 b_{n}, \quad b_{n+1}-b_{n}=2 a_{n}
$$

and hence

$$
a_{n+2}-2 a_{n+1}+5 a_{n}=0, \quad b_{n+2}-2 b_{n+1}+5 b_{n}=0 .
$$

With $a(x), b(x)$ the ordinary generating functions for $a_{n}, b_{n}$, these recurrences and the boundary conditions $a_{0}=b_{0}=1, a_{1}=-1, b_{1}=3$ imply

$$
\left(1-2 x+5 x^{2}\right) a(x)=1-3 x, \quad\left(1-2 x+5 x^{2}\right) b(x)=1+x .
$$

The first few values of the numbers $a_{n}, b_{n}$ are

\begin{tabular}{rrrrrrrrrr}
\hline$n$ & 0 & 1 & 2 & 3 & 4 & 5 & 6 & 7 & 8 \\
\hline$a_{n}$ & 1 & -1 & -7 & -9 & 17 & 79 & 73 & -249 & -863 \\
$b_{n}$ & 1 & 3 & 1 & -13 & -31 & 3 & 161 & 307 & -191
\end{tabular}

Further, since $S_{0}(x)=S_{1}(x)=1$, Touchard's relation entails

$$
2-S(i, y)=1-y-(1+i) y^{2}(1-(1+2 i) y)^{-1}
$$

$$
=\left(1-(2+2 i) y+i y^{2}\right)(1-(1+2 i) y)^{-1} \text {. }
$$

Then by (3a)

$$
T(i, y)=(1-(1+2 i) y)\left(1-(2+2 i) y+i y^{2}\right)^{-1} .
$$

Noting that $1-2(1+i) y+i y^{2}=(1-(1+i) y)^{2}-i y^{2}$, (11) may be expanded as follows

$$
\begin{aligned}
T(i, y) & =[1-(1+2 i) y] \sum_{j=0} i^{j} y^{2 j}(1-(1+i) y)^{-2 j-2} \\
& =[1-(1+2 i) y] \sum_{j=0} \sum_{k=0}\left(\begin{array}{c}
2 j+k+1 \\
k
\end{array}\right) i^{j}(i+1)^{k} y^{2 j+k} \\
& =\sum_{n=0} y^{n}(1+i)^{n}\left(f_{n}-\frac{3+i}{2} f_{n-1}\right)
\end{aligned}
$$


with $f_{n}=\Sigma_{0}\left(\begin{array}{c}n+1 \\ 2 j+1\end{array}\right) 2^{-j}$. Thus

$$
T_{n}(i)=1 / 2(1+i)^{n}\left(2 f_{n}-(3+i) f_{n-1}\right), \quad n=0,1,2, \cdots .
$$

The recurrence for $f_{n}$, following from that for binomial coefficients, is $f_{n}-2 f_{n-1}+$ $1 / 2 f_{n-2}=\delta_{n 0}$. Hence its generating function is $f(x)=\left(1-2 x+x^{2} / 2\right)^{-1}$ and the partial fraction expansion of the latter shows that

$$
f_{n}=\alpha\left[(1+\alpha)^{n+1}-(1-\alpha)^{n+1}\right], \quad \alpha=2^{-1 / 2} .
$$

Use of the recurrence in (12) provides the alternate form

$$
T_{n}(i)=1 / 2(1+i)^{n}\left[(1-i) f_{n-1}-f_{n-2}\right], \quad n=1,2, \cdots,
$$

and hence

$$
\begin{aligned}
t_{n}(i) & =(1-i)^{n} T_{n}(i)=2^{n-1}\left(2 f_{n}-(3+i) f_{n-1}\right) \\
& =2^{n-1}\left[(1-i) f_{n-1}-f_{n-2}\right], \quad n=1,2, \cdots .
\end{aligned}
$$

Values of $2^{n-1} f_{n}=g_{n}$ and the real and imaginary parts of $t_{n}(i)$, designated $R t_{n}(i)$ and $I t_{n}(i)$ follow

\begin{tabular}{crrrrrrrrrr}
\hline$n$ & 0 & 1 & 2 & 3 & 4 & 5 & 6 & 7 & 8 & 9 \\
\hline$g_{n}$ & 1 & 2 & 7 & 24 & 82 & 280 & 956 & 3264 & 11144 & 38048 \\
$R t_{n}(i)$ & 1 & 1 & 2 & 6 & 20 & 68 & 232 & 792 & 2704 & 9232 \\
$I t_{n}(i)$ & 0 & -1 & -4 & -14 & -48 & -164 & -580 & -1912 & -6528 & -22288
\end{tabular}

For verification of the table, it may be noted that

$$
t(i, y)=T(i,(1-i) y)=[1-(3+i) y]\left[1-4 y+2 y^{2}\right]^{-1} .
$$

Hence

$$
t_{n}(i)-4 t_{n-1}(i)+2 t_{n-2}(i)=\delta_{n 0}-(3+i) \delta_{n 1}
$$

or

$$
t_{n}(i)=4 t_{n-1}(i)-2 t_{n-2}(i), \quad n=2,3, \cdots
$$

For a similar study of the real and imaginary parts of $T_{n}(i)$ it is convenient to ignore the initial values $T_{0}(i)=T_{1}(i)=1$ and define

$$
T_{n+2}(i)=A_{n}+i B_{n}, \quad n=0,1, \cdots ; i^{2}=-1 .
$$

Then with $A(y), B(y)$ the generating functions of $A_{n}, B_{n}$,

$$
y^{-2}[T(i, y)-1-y]=A(y)+i B(y)
$$

using Eq. (11), it is found that

$$
[2+i(1-y)]\left[1-2 y i\left(2 y-y^{2}\right)\right]^{-1}=A(y)+i B(y) .
$$


With $D(y)=1-4 y+8 y^{2}-4 y^{3}+y^{4}$, it turns out that

$$
D(y) A(y)=2-6 y+3 y^{2}-y^{3}, \quad D(y) B(y)=1+y .
$$

The corresponding recurrences are

$$
\begin{aligned}
& A_{n}-4 A_{n-1}+8 A_{n-2}-4 A_{n-3}+A_{n-4}=2 \delta_{n 0}-6 \delta_{n 1}+3 \delta_{n 2}-\delta_{n 3} \\
& B_{n}-4 B_{n-1}+8 B_{n-2}-4 B_{n-3}+B_{n-4}=\delta_{n 0}+\delta_{n 1} .
\end{aligned}
$$

Notice that the right-hand side is zero for $n>3$ in the first, $n>1$ in the second.

An immediate consequence of (11) is the recurrence

$$
T_{n}(i)-(2+2 i) T_{n-1}(i)+i T_{n-2}(i)=\delta_{n 0}-(1+2 i) \delta_{n 1} .
$$

Using (16) in this equation (with $n$ replaced by $n+2$ ) and separating real and imaginary parts, it follows that

$$
A_{n+2}-2 A_{n+1}=-2 B_{n+1}+B_{n}, \quad B_{n+2}-2 B_{n+1}=2 A_{n+1}-A_{n} .
$$

Hence

$$
\begin{aligned}
& A_{n+2}-A_{n}=B_{n+2}-4 B_{n+1}+B_{n}, \\
& A_{n+4}-A_{n}=B_{n+4}-4 B_{n+3}+2 B_{n+2}-4 B_{n+1}+B_{n}=-6 B_{n+2},
\end{aligned}
$$

the last step by the second recurrence in (19). Similarly, it is found that

$$
B_{n+4}-B_{n}=-\left(A_{n+4}-4 A_{n+3}+2 A_{n+2}-4 A_{n+1}+A_{n}\right)=6 A_{n+2} .
$$

Combination of (22) and (23) produces

$$
A_{n+8}+34 A_{n+4}+A_{n}=0, \quad B_{n+8}+34 B_{n+4}+B_{n}=0 .
$$

Illustrative numerical results are in the following table

\begin{tabular}{rrrrrrrrrrrrr}
\hline$n$ & 0 & 1 & 2 & 3 & \multicolumn{1}{c}{4} & \multicolumn{1}{c}{5} & \multicolumn{1}{c}{6} & \multicolumn{1}{c}{7} & \multicolumn{1}{c}{8} & 9 & 10 & 11 \\
\hline$A_{n}$ & 2 & 2 & -5 & -29 & -70 & -70 & 169 & 985 & 2378 & 2378 & -5741 & -33461 \\
$B_{n}$ & 1 & 5 & 12 & 12 & -29 & -169 & -408 & -408 & 985 & 5741 & 13860 & 13860
\end{tabular}

I do not take space to prove the identities apparent in the table, namely: $A_{4 n}=$ $A_{4 n+1}, B_{4 n+2}=B_{4 n+3}, A_{4 n+2}=-B_{4 n+1}, A_{4 n+3}=B_{4 n+4}$.

Department of Mathematics

Rockefeller University

New York, New York 10021

1. A. ERrera, “Une problème d'énumeration," Mém. Acad. Roy. Belgique Coll. $8^{0}$, (2), v. $11,1931,26 \mathrm{pp}$.

2. M. KAC \& J. C. WARD, "A combinatorial solution of the two-dimensional Ising model," Phys. Rev., v. 88, 1952, pp. 1332-1337.

3. C. L. MALLOWS \& JOHN RIORDAN, "The inversion enumerator for labeled trees," Bull. Amer. Math. Soc.,v. 74, 1968, pp. 92-94. MR 36 \#1341. 
4. JOHN RIORDAN, Combinatorial Identities, Wiley, New York, 1968. MR 38 \#53.

5. JOHN RIORDAN, "Ballots and trees," J. Combinatorial Theory, v. 6, 1969, pp. 408411. MR $38 \# 3157$.

6. JACQUES TOUCHARD, "Contribution à l'étude du problème des timbres poste," Canad. J. Math., v. 2, 1950, pp. 385-398. MR 12, 312.

7. JACQUES TOUCHARD, "Sur une problème de configurations," C. R. Acad. Sci. Paris, v. 230, 1950, pp. 1997-1998. MR 12, 44.

8. JACQUES TOUCHARD, "Sur une problème de configurations et sur les fractions continues," Canad. J. Math., v. 4, 1952, pp. 2-25. MR 13, 716.

9. A. M. JAGLOM \& I. M. JAGLOM, Non-Elementary Problems in an Elementary Exposition. Vol. I, GITTL, Moscow, 1954; English transl., Challenging Mathematical Problems With Elementary Solutions. Vol. I: Combinatorial Analysis and Probability Theory, Holden-Day, San Francisco, Calif., 1964. MR 17, 18; 29 \#2193. 